\title{
Blind Source Separation Based on Rotation of Joint Distribution Without Inversion of Positive and Negative Sign
}

\author{
Masato Iikawa $^{\mathrm{a}, *}$, Takaaki Ishibashi ${ }^{\mathrm{a}}$ \\ ${ }^{a}$ Department of Information, Communication and Electronic Engineering, \\ National Institute of Technology, Kumamoto College \\ 2659-2 Suya, Koshi, Kumamoto 861-1102, Japan \\ *Corresponding Author: te14iikawa@g.kumamoto-nct.ac.jp
}

\begin{abstract}
This paper proposes a blind source separation method based on rotation of a joint distribution of observed mixture signals with microphones. Our previous method can separate the sound sources using only the information of observed signals. The separation method has sign inversion problem in some cases. The problem means that the positive and negative signs of the estimated signal are inverted. In the case of using the signal from the start to the end of the human speech, the sign of the estimated signal is not a big problem. However, in the case of short-time frame processing, inversion of a sign at the junction point of the separated signal becomes a big problem. Therefore, we propose a new separation method without indeterminacy of positive and negative sign of the estimated signal.
\end{abstract}

Keywords: blind source separation, joint distribution, rotation BSS.

\section{Introduction}

In order to estimate sound sources from observed mixture signals without using the information about source signals and transfer functions, many BSS (Blind Source Separation) methods have proposed. For BSS, ICA (Independent Component Analysis) can estimate original source signals from their mixtures, provided that the sources are statistically independent. For the instantaneous mixtures, the original sources can be completely recovered except for indeterminacy of scale and permutation $^{(1,2,3)}$. The indeterminacy of scale is that the amplitude scale of the separated signals is not equal to that of the source signals. The indeterminacy of permutation is that the order of the separated signals is not equal to that of the source signals. Furthermore, ICA algorithms are iteration methods based on the gradient method or the Newton method. This fact means that these algorithms are not good at a real-time processing.

For a real-time separation, several methods have been proposed. SS (Spectral Subtraction) ${ }^{(4)}$, SAFIA (sound source Segregation based on estimating incident Angle of each Frequency component of Input signals Acquired by multiple microphones) ${ }^{(5)}$ and and NMF (Nonnegative Matrix

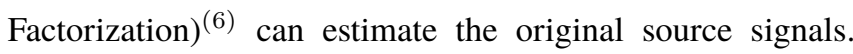
In these methods, the musical-noise has been generated depending on the parameter. In order to reduce the musicalnoise, a method based on the high-order statistics has been $\operatorname{proposed}^{(7)}$. However, multivariate data are necessary for the method.

The authors have already proposed a separation method based on the rotation of the distribution of the observed signals $^{(8)}$. The previous methods can estimate the original source signals. However the estimated signals using the previous method have sign indeterminacy problem in some cases. Therefore, the paper proposes a new separation method without inversion of positive and negative sign of the estimated signal.

\section{Observed mixing model}

Consider that some sound sources $s_{n}(t)(n=1,2, \cdots, N)$ are observed with some microphones, the observed mixture signals $x_{m}(t)(m=1,2, \cdots, M)$ are expressed as

$$
x_{m}(t)=\sum_{n=1}^{N} a_{m n} s_{n}(t)
$$

where $a_{m n}$ denotes a mixing parameter, $t$ denotes index of time series, $N$ and $M$ are the number of sound sources and mixture signals, respectively. 

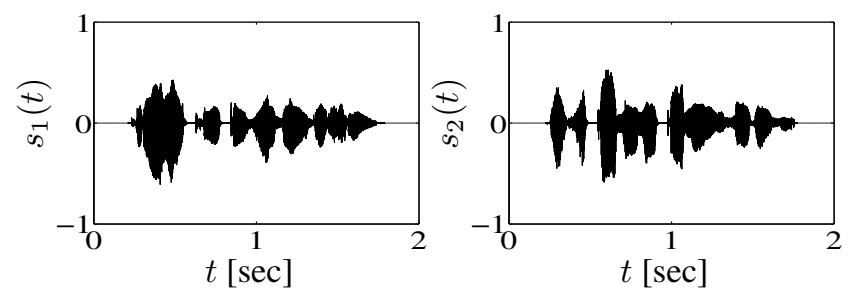

Fig. 1: Waveforms of the sound source signals.

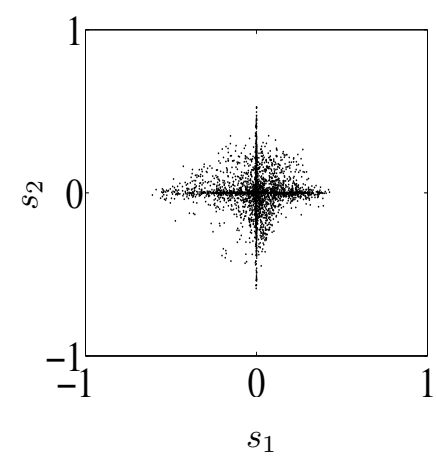

Fig. 2: Distribution of the sound source signals.

In a real environment, the signals observed at microphones are not instantaneous mixtures but are convoluted version of the sound sources as

$$
x_{m}(t)=\sum_{n=1}^{N} \sum_{\tau} a_{m n}(\tau) s_{n}(t-\tau)
$$

where $a_{m n}(\tau)$ denotes transfer functions from the $n$-th sources to the $m$-th microphones. The mixtures $x_{m}(t)$ are transformed into the short time spectra by the discrete Fourier transform to process in frequency domain as follows.

$$
x_{m}(\omega, k)=\sum_{t} e^{-j \omega t} x_{m}(t) w(t-k \tau)
$$

where $\omega$ denotes a frequency, $k$ the frame number, $\tau$ the frame shift time and $w(t)$ a window function. In the frequency domain, the mixtures are approximated as follows.

$$
\boldsymbol{x}(\omega, k)=\sum_{n=1}^{N} a_{m n}(\omega) s_{n}(\omega, k)
$$

By expressing as Eq (4), the convolution mixing process can be thought of as an instantaneous mixing process. On account of this, there have been reported many trials to separate the convoluted mixtures in the frequency domain. In the following, we discuss in the instantaneous mixture model for simplicity.
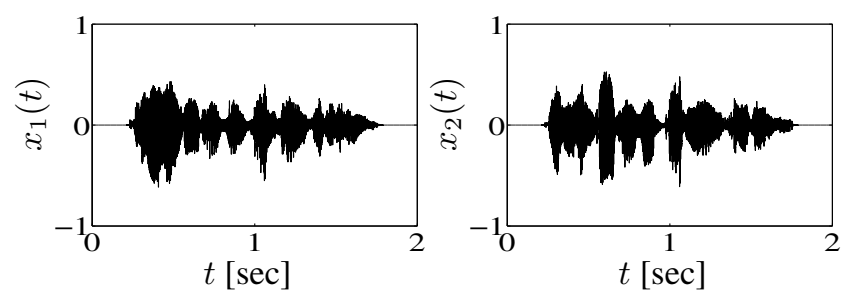

Fig. 3: Waveforms of the observed mixture signals.

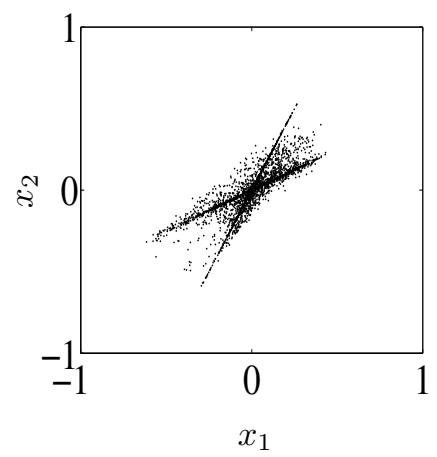

Fig. 4: Distribution of the observed mixture signals.

\section{BSS Based on Rotation of Distribution}

\subsection{Principle of Rotation BSS}

In order to estimate the source signals, we have already proposed a BSS method based on the rotation of the joint distribution of the observed signals.

Consider that two speakers have uttered in front of two microphones. These waveforms are shown in Fig. 1. Using these waveforms, their joint distribution is shown in Fig. 2 where the horizontal and the vertical axis are denoted by the amplitude of $s_{1}(t)$ and $s_{2}(t)$, respectively.

The mixture signals $x_{m}(t)$ are observed by Eq (1). The waveforms and the joint distribution of $x_{m}(t)$ are shown in Fig. 3 and Fig. 4, respectively. From these facts, the essence of the BSS is to transform from the distribution of the mixtures as shown in Fig. 4 to the distribution of the sources as shown in Fig. 2.

Our basic rotation BSS method based on whitening and rotation. In order to orthogonalize of the crossed distribution of Fig. 4, we calculate as

$$
\widetilde{\boldsymbol{x}}(t)=\Lambda^{-\frac{1}{2}} \Phi^{T} \boldsymbol{x}(t)=Q \boldsymbol{x}(t)
$$

where $\boldsymbol{x}(t)=\left[x_{1}(t), \cdots, x_{m}(t), \cdots, x_{M}(t)\right]^{T}$ denotes the mixture signals, $\Phi$ is the orthogonal matrix of eigenvectors of $E\left[\boldsymbol{x} \boldsymbol{x}^{T}\right], \Lambda$ is the diagonal matrix of its eigenvalues and $Q$ denotes a whitening matrix. Fig. 5 shows the joint distribution 


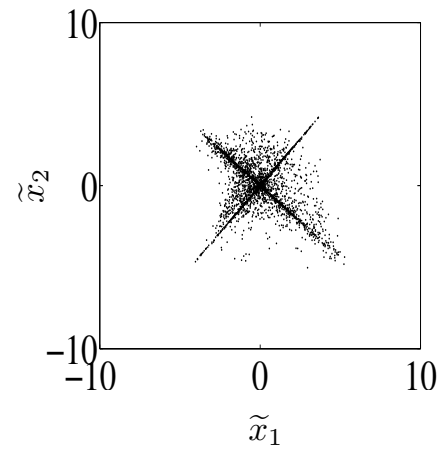

Fig. 5: Distribution of the whitened mixture signals.

of the whitened mixture signals $\widetilde{\boldsymbol{x}}(t)$. From the figure, it is found that the joint distribution of the sources is recovered except for indeterminacy of rotation.

To solve the indeterminacy of rotation, we calculate the angle for the points of the joint distribution of $\widetilde{\boldsymbol{x}}(t)$ as

$$
\phi(t)=\tan ^{-1} \frac{\widetilde{x}_{2}(t)}{\widetilde{x}_{1}(t)},
$$

and obtain the direction histogram of $\phi(t)$. The rotation angle $\theta$ is estimated as

$$
\theta=\arg \max _{\phi(t)} \operatorname{hist}(\phi(t))
$$

and we estimate the rotation matrix $R$ as follows.

$R=\left[\begin{array}{cc}\cos (-\theta) & -\sin (-\theta) \\ \sin (-\theta) & \cos (-\theta)\end{array}\right]=\left[\begin{array}{cc}\cos \theta & \sin \theta \\ -\sin \theta & \cos \theta\end{array}\right]$

In the case which the number of the source signals is three or more, a histogram is calculated from the observed signals by multi microphones. And a joint distribution with a multidimensional space is orthogonalized based on the rotation angle from the histogram.

Therefore, the rotation BSS method as follows.

$$
\boldsymbol{y}(t)=R Q \boldsymbol{x}(t)
$$

where $\boldsymbol{y}(t)=\left[y_{1}(t), \cdots, y_{n}(t), \cdots, y_{N}(t)\right]^{T}$ denotes the estimated separating signals. Using the method, the rotated joint distribution and the rotated source signals show in Fig. 6 and Fig. 7, respectively. In these figures, the rotated distribution and signals are recovered the sources except for the scaling indeterminacy.

\subsection{Rotation BSS Without Whitening}

The basic rotation BSS can estimate the source signals. However, the estimated signals have a scaling indeterminacy. Furthermore, when the dimension of the distribution is changed by increasing or decreasing the number of

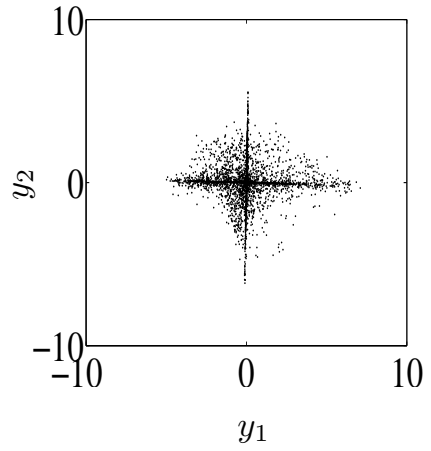

Fig. 6: Distribution of the separated signals.
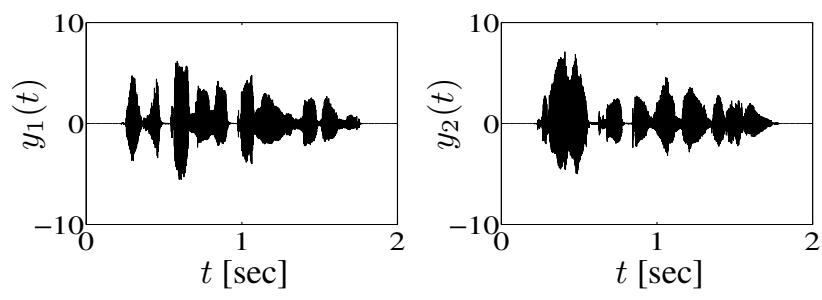

Fig. 7: Waveforms of the separated signals.

sound sources, the algorithm is complicated because it requires whitening. Therefore, a rotation BSS algorithm without whitening has proposed ${ }^{(8)}$.

In the same way as Eq (6), we calculate the angle without whitening by using observed mixture signals $x_{m}(t)$ in the condition of two microphones as follows.

$$
\phi(t)=\tan ^{-1} \frac{x_{2}(t)}{x_{1}(t)}
$$

The rotation angle $\theta_{n}$ of $n$-th peak of the histogram is estimated as Eq (7). Therefore, the rotation BSS without whitening has proposed as follows.

$$
y_{n}(t)=x_{2}(t) \cos \theta_{n}-x_{1}(t) \sin \theta_{n}
$$

By detecting the peaks of the histogram, the method can remove the signal corresponding to the peak. In other words, the method founds the direction of the source signal to be deleted and estimates another source signal.

The rotation BSS method can estimate the original source signals without the scaling indeterminacy. However, the estimated signals have the indeterminacy of sign. It means that the positive and negative signs of the estimated signal are inverted in some cases. Using the speech signal from the start to the end, the sign of the estimated signal is not a big problem. However, in the case of short-time frame processing, inversion of sign at the junction point of the separated signal 
becomes a big problem. Therefore, it is necessary to solve the sign problem.

\section{Variable Arbitrary Directivity Pattern}

In this section, we propose a new separation method without inversion of positive and negative sign of the estimated signal. In the above method, the rotation angle $\theta_{n}$ depends only on the sound component to be deleted. It means that the information of the estimating signal to be extracted is not used. Therefore, there is a problem that the sign of the estimated signal is reversed in the case of reversing the rotation.

The cause of the indeterminacy of the sign is that the $\theta_{n}$ is larger than the other one. Therefore, all the rotation angles are estimated, and the sign problem is solved based on the magnitude relation of the angles. When the angle used for estimation is smaller than the other one, the estimation process uses Eq (11). In the case which the angle used for estimation is larger than the other one, the sign problem can be solved by multiplying the estimated signal of $\mathrm{Eq}(11)$ by -1 .

The rotation BSS without the sign indeterminacy in the case of two sound sources is proposed as follows.

$$
\begin{aligned}
& y_{1}(t)= \begin{cases}x_{2}(t) \cos \theta_{1}-x_{1}(t) \sin \theta_{1} & \left(\text { if } \theta_{1}<\theta_{2}\right) \\
x_{1}(t) \sin \theta_{1}-x_{2}(t) \cos \theta_{1} & \left(\text { if } \theta_{1}>\theta_{2}\right)\end{cases} \\
& y_{2}(t)= \begin{cases}x_{2}(t) \cos \theta_{2}-x_{1}(t) \sin \theta_{2} & \left(\text { if } \theta_{1}>\theta_{2}\right) \\
x_{1}(t) \sin \theta_{2}-x_{2}(t) \cos \theta_{2} & \left(\text { if } \theta_{1}<\theta_{2}\right)\end{cases}
\end{aligned}
$$

The estimation signal using the proposed method has no indeterminacy of the sign.

\section{Simulation}

In order to verify our proposals, several simulations were carried out. The target source signal $s_{1}(t)$ was $6[\mathrm{sec}]$ human speech signal in the database ${ }^{(9)}$. The noise source $s_{2}(t)$ was $6[\mathrm{sec}]$ another human speech. These signals were sampled at the rate of $8[\mathrm{kHz}]$ with 16 [bit] resolution. Using these sources, the mixture signals are generated by Eq (1) with the mixing parameters $a_{m n}=0.9 \pm \eta(m=n)$ and $a_{m n}=$ $0.6 \pm \eta(m \neq n), \eta$ denotes a random value from 0 to 0.1 . Under this condition, 30 mixture signals patterns (permutation of 3 males and 3 females ${ }_{6} \mathrm{P}_{2}$ ) were generated. We confirmed that the method can estimate the source signals without the sign indeterminacy in all data.

\section{Conclusion}

This paper proposes the rotation BSS method without inversion of positive and negative sign of the estimated signal. Using the joint distribution of the observed mixture signals, the proposed method estimates the source signals based on detecting the peaks components of the histogram of the distribution. Furthermore, the method is solved by inverting the sign in the case of reversing rotation. From the simulation, it is found that the method can estimate the source signals without the sign indeterminacy in all data.

\section{Acknowledgment}

This work was supported by JSPS KAKENHI Grant Number $16 \mathrm{~K} 21583$.

\section{References}

(1) A. Hyvärinen, J. Karhunen, and E. Oja: "Independent component analysis", John Wiley \& Sons, Ltd, 1998

(2) A. Cichocki and S. Amari: "Adaptive blind signal and image processing, learning algorithm and applications", John Wiley \& Sons, Ltd, 2002

(3) S. Makino, T.-W. Lee and H. Sawada: "Blind speech separation”, Springer, 2007

(4) S. F. Boll: "Suppression of acoustic noise in speech using spectral subtraction", IEEE Transactions on Acoustics, Speech and Signal Processing, Vol. ASSP-27, No. 2, pp. 113-120, 1979

(5) M. Aoki, M. Okamoto, S. Aoki, H. Matsui, T. Sakurai and Y. Kaneda: "Sound source segregation based on estimating incident angle of each frequency component of input signals acquired by multiple microphones, Acoustical Science and Technology", Vol. 22, No. 2, pp. 149-157, 2001

(6) A. Cichocki, R. Zdunek and S. Amari: "Nonnegative matrix and tensor factorization", IEEE Signal Processing Magazine, Vol. 25, No. 1, pp. 142-145, 2008

(7) R. Miyazaki, H. Saruwatari, S. Nakamura, K. Shikano, K. Kondo, J. Blanchette, and M. Bouchard: "Musicalnoise-free blind speech extraction integrating microphone array and iterative spectralsSubtraction," Signal Processing, Elsevier, Vol. 102, pp. 226-239, 2014

(8) K. Higuchi, C. Okuma and T. Ishibashi: “ Blind source separation and human speech extraction for three sound sources using silent interval," The 5th IIAE International Conference on Intelligent Systems and Image Processing 2017, pp. 205-208, 2017

(9) Acoustical Society of Japan: "ASJ continuous speech corpus Japanese newspaper article sentences", JNAS Vols. 1-16, 1997 